\title{
Correction to: HYPE: Predicting Blood Pressure from Photoplethysmograms in a Hypertensive Population
}

Ariane Morassi Sasso (D), Suparno Datta (D), Michael Jeitler (D), Nico Steckhan (D), Christian S. Kessler (D), Andreas Michalsen, Bert Arnrich, and Erwin Böttinger

\section{Correction to:}

\section{Chapter "HYPE: Predicting Blood Pressure}

from Photoplethysmograms in a Hypertensive Population" in: M. Michalowski and R. Moskovitch (Eds.): Artificial Intelligence in Medicine, LNAI 12299, https://doi.org/10.1007/978-3-030-59137-3_29

The original version of this chapter was revised. The conclusion section was corrected and reference was added. 